\title{
SARS-CoV-2 antibody assay after vaccination: one size does not fit all
}

https://doi.org/10.1515/cclm-2021-0703

Received June 16, 2021; accepted June 18, 2021;

published online June 30, 2021

Keywords: antibodies; Comirnaty Biontech/Pfizer; SARS-CoV-2; SARS-CoV-2 serology; serology; vaccination; vaccine.

To the Editor,

We read with interest the article of Salvagno and colleagues on anti-SARS-CoV-2 antibodies response after BNT162b2 (Comirnaty Biontech/Pfizer) COVID-19 (coronavirus disease 2019) vaccination, which appears in the current issue of the Journal [1]. The Authors are to be congratulated for the valuable results reported, and for their considerations regarding our previous paper [2]. Salvagno and colleagues measured anti-SARS-CoV-2 S-RBD IgG serum levels in 194 healthcare workers who received a complete BNT162b2 COVID-19 vaccine cycle (prime and boost inoculums). Anti-SARS-CoV-2 S-RBD immunoglobulin $\mathrm{G}(\mathrm{IgG})$ antibodies were measured at baseline (T0, before the first vaccination), at 21 days (T1) and 51 days (T2) after the first vaccine dose (i.e. immediately before the second vaccine dose and 30 days after the second). Among the individuals included in the study, $15.5 \% \quad(\mathrm{n}=30)$ had elevated anti-SARS-CoV-2 S-RBD IgG levels at baseline, and were considered "baseline positive".

The first important aspect to consider is the comparison between anti-SARS-CoV-2 S-RBD IgG values

\footnotetext{
*Corresponding author: Mario Plebani, Department of MedicineDIMED, University of Padova, Padova, Italy; and Department of Laboratory Medicine, University-Hospital of Padova, Padova, Italy, E-mail: mario.plebani@unipd.it. https://orcid.org/0000-00020270-1711

Chiara Cosma, Department of Laboratory Medicine, UniversityHospital of Padova, Padova, Italy

Andrea Padoan, Department of Medicine-DIMED, University of Padova, Padova, Italy; and Department of Laboratory Medicine, University-Hospital of Padova, Padova, Italy. https://orcid.org/00000003-1284-7885
}

found at $\mathrm{T} 1$ for individuals $\mathrm{Ab}$ positive at baseline (74 kU/L) and at T2 for those who were seronegative at baseline $(513 \mathrm{kU} / \mathrm{L})$. These values differ considerably from findings reported in our study [2] using the same immunoassay, in which the first Pfizer BNT162b2 administration was effective in achieving antibodies levels comparable to those of the $\mathrm{T} 2$ of infectious-naïve subjects. Other studies in the literature describe that people with a prior SARS-CoV-2 infection have IgG levels similar to those observed in infectious-naïve individuals after the second vaccine dose [3]. The many possible explanations for these discrepancies include differences between the populations tested, degree of SARS-CoV-2 $\mathrm{Ab}$ developed during the first vaccine inoculum (the amount of specific antibodies detectable at the time of the first dose), and heterogeneity of immune response to previous SARS-CoV-2 infection. Another explanation might be the timing of sample collection after the first inoculum, as a recent paper from the same Authors has shown a rapid decrease of anti-SARS-CoV-2 S-RBD IgG in a time-kinetics case-series study [4]. More generally, this heterogeneity appears to support the hypothesis that, even if SARS-CoV-2 antibody tests cannot be recommended in the population at large, specific groups of individuals might benefit from testing, especially for ruling out a lack of immune response after vaccination $[5,6]$. For example, physicians, patients and their families are concerned about the immune response after COVID-19 vaccine in cancer patients under treatment [5]. Other categories with reduced immune response to COVID-19 vaccines are patients on treatment for chronic inflammatory conditions and immunosuppressive therapy $[6,7]$. Strategies for improving the immunogenicity of currently developed vaccines, and for allowing a personalized serology-based approach, have been reported and discussed in the literature, though further research on this issue would still be needed [5].

The second point regards the lack of correlation between anti-SARS-CoV-2S-RBD IgG values and demographic variables, namely age and gender. Indeed, the response to vaccination is often found to be lower in elderly adults than in young individuals, such reduced immunogenicity being 
attributed to adaptive immunosenescence [8]. In a recent study by Muller et al. [8], 176 volunteers were analyzed for vaccine-induced SARS-CoV-2 spike specific IgG after a prime and boost vaccination campaign using BNT162b2 in individuals over 80 years of age compared to those aged 60 years or younger. The Authors found that while the majority of both young and elderly vaccine recipients displayed augmented IgG responses after their second vaccination, values for induction of IgG and neutralizing antibodies were significantly lower in the elderly subjects. These data confirm that even if an antibody response is present in all individuals, there are some differences between age-specific groups, especially in subjects over 80 years of age. In a recent study by Pellini et al. [9], women were found to generate higher antibodies levels than men in multivariate analyses. Further data should hence be generated to provide more robust evidence of the kinetics and time-dependent reduction of SARS-CoV-2 antibodies after vaccination. Moreover, additional efforts shall also be made for improving harmonization, standardization and comparability of anti-SARS-CoV-2 serology, although a tentative effort for harmonizing different SARS-CoV-2 assays cut-offs has been recently undertaken [10].

The clinical efficacy of vaccination can be measured not only on the basis of ability to prevent infection, but also ability to reduce symptoms, hospitalization and death. At this stage in the pandemic, it has been postulated that vaccination effectively paves the way for generating durable, sound herd immunity. However, a body of evidence underpins the importance of evaluating serology in assessing the immunogenic response of vaccinated individuals, especially in specific groups of individuals with a compromised immune response [11].

Research funding: None declared.

Author contributions: All authors have accepted responsibility for the entire content of this manuscript and approved its submission.

Competing interests: Authors state no conflict of interest. Informed consent: Informed consent was obtained from all individuals included in this study.

Ethical approval: Not applicable.

\section{References}

1. Salvagno G, Henry BM, Pighi L, Nitto SD, Gianfilippi GL, Lippi G. Monitoring of the immunogenic response to Pfizer BNT162b2 mRNA COVID-19 vaccination in healthcare workers with Snibe SARS-CoV-2 S-RBD IgG chemiluminescent immunoassay. Clin Chem Lab Med 2021;59:e377-9.

2. Padoan A, Dall'Olmo L, Barbaro F, Cosma C, Basso D, Cattelan A, et al. Antibody response to first and second dose of BNT162 b2 in a cohort of characterized healthcare workers. Clin Chim Acta 2021; 519:60-3.

3. Ebinger JE, Fert-Bober J, Printsev I, Wu M, Sun N, Prostko JC, et al. Antibody responses to the BNT162b2 mRNA vaccine in individuals previously infected with SARS-CoV-2. Nat Med 2021. https://doi. org/10.1038/s41591-021-01325-6 [Epub ahead of print].

4. Danese E, Montagnana M, Salvagno GL, Peserico D, Pighi L, de Nitto $S$, et al. Comprehensive assessment of humoral response after Pfizer BNT162b2 mRNA Covid-19 vaccination: a three-case series. Clin Chem Lab Med 2021;59:1585-91.

5. Massarweh A, Eliakim-Raz N, Stemmer A, Levy-Barda A, Yust-Katz S, Zer A, et al. Evaluation of seropositivity following BNT162b2 messenger RNA vaccination for SARS-CoV-2 in patients undergoing treatment for cancer. JAMA Oncol 2021:1-8. https:// doi.org/10.1001/jamaoncol.2021.2155.

6. Havlin J, Svorcova M, Dvorackova DE, Lastovicka DJ, Lischke R, Kalina APT, et al. Immunogenicity of BNT162b2 mRNA COVID-19 vaccine and SARS-CoV-2 infection in lung transplant recipients. J Heart Lung Transplant 2021:4-8. https://doi.org/10.1016/j. healun.2021.05.004.

7. Geisen UM, Berner DK, Tran F, Sümbül M, Vultriede L, Ciripoi M, et al. Immunogenicity and safety of anti-SARS-CoV-2 mRNA vaccines in patients with chronic inflammatory conditions and immunosuppressive therapy in a monocentric cohort. Ann Rheum Dis 2021:1-6.

8. Muller L, Andreée M, Moskorz W, Drexler I, Walotka L, Grothmann R, et al. Age-dependent immune response to the Biontech/Pfizer BNT162b2 COVID-19 vaccination. Clin Infect Dis 2021. https://doi.org/10.1093/cid/ciab381 [Epub ahead of print].

9. Pellini R, Venuti A, Pimpinelli F, Abril E, Blandino G, Campo F, et al. Initial observations on age, gender, BMI and hypertension in antibody responses to SARS-CoV-2 BNT162b2 vaccine. EClinicalMedicine 2021. https://doi.org/10.1016/j.eclinm.2021. 100928 [Epub ahead of print].

10. Plebani M, Padoan A, Negrini D, Carpinteri B, Sciacovelli L. Diagnostic performances and thresholds: the key to harmonization in serological SARS-CoV-2 assays? Clin Chim Acta 2020;509:1-7.

11. Lippi G, Henry BM, Plebani M. Anti-SARS-CoV-2 antibodies testing in recipients of COVID-19 vaccination: Why, When, and How? Diagnostics 2021;11:941. 